\title{
The influence of a blended, theoretically- informed pre-implementation strategy on school-based clinician implementation of an evidence-based trauma intervention
}

Aaron R. Lyon ${ }^{1 *}$ D, Clayton R. Cook ${ }^{2}$, Mylien T. Duong ${ }^{1,3}$, Semret Nicodimos ${ }^{1}$, Michael D. Pullmann ${ }^{1}$, Stephanie K. Brewer ${ }^{1}$, Larissa M. Gaias ${ }^{1}$ and Shanon Cox ${ }^{1}$

\begin{abstract}
Background: Individual-level implementation determinants, such as clinician attitudes, commonly influence the successful adoption of evidence-based practices, but few explicit strategies have been tested with regard to their ability to impact these key mechanisms of change. This paper reports on an initial test of a blended, theoretically informed pre-implementation strategy designed to target malleable individual-level determinants of behavior change. Beliefs and Attitudes for Successful Implementation in Schools (BASIS) is a brief and pragmatic preimplementation strategy that uses strategic education, social influence techniques, and group-based motivational interviewing to target implementation attitudes, perceived social norms, perceived behavioral control, and behavioral intentions to implement among mental health clinicians working in the education sector.
\end{abstract}

Methods: As part of a pilot trial, 25 school mental health clinicians were randomized to BASIS $(n=12)$ or an attention control placebo $(n=13)$, with both conditions receiving training and consultation in an evidence-based intervention for youth experiencing trauma (the Cognitive Behavioral Intervention for Trauma in Schools). Theorized mechanisms of change (attitudes, perceived social norms, perceived behavioral control, and behavioral intentions) were assessed at baseline, post-training, and 4-month follow-up. Clinician participation in post-training consultation and intervention adoption were also tracked.

Results: A series of regression models and independent sample $t$ tests indicated that BASIS had significant, medium to large effects on the majority of its proximal mechanisms from baseline to post-training. BASIS was also associated with a greater latency between initial training in the intervention and discontinuation of participation in post-training consultation, with clinicians in the BASIS condition persisting in consultation for an average of 134 days versus 32 days for controls, but this difference was not statistically significant. At 4-month follow-up, most differences in the theorized mechanisms had attenuated, and approximately the same small number of BASIS clinicians adopted the trauma intervention as controls.

Conclusion: Findings suggest that the brief BASIS pre-implementation strategy had a significant influence on its proximal mechanisms of change, but that these changes did not persist over time or translate into adoption of the trauma intervention. Implications for theory refinement, revisions to the BASIS protocol, and next steps for research surrounding individual-level implementation strategies are discussed.

(Continued on next page)

\footnotetext{
* Correspondence: lyona@uw.edu

${ }^{1}$ University of Washington, 6200 NE 74th Street, Suite 100, Seattle, WA 98115,

USA

Full list of author information is available at the end of the article
}

(c) The Author(s). 2019 Open Access This article is distributed under the terms of the Creative Commons Attribution 4.0 International License (http://creativecommons.org/licenses/by/4.0/), which permits unrestricted use, distribution, and reproduction in any medium, provided you give appropriate credit to the original author(s) and the source, provide a link to the Creative Commons license, and indicate if changes were made. The Creative Commons Public Domain Dedication waiver (http://creativecommons.org/publicdomain/zero/1.0/) applies to the data made available in this article, unless otherwise stated. 
(Continued from previous page)

Trial registration: ClinicalTrials.gov Identifier: NCT03791281. Registered 31 December 2018—Retrospectively registered

Keywords: Individual determinants, Implementation strategy, Theory of planned behavior, Trauma intervention, Behavioral intentions, Adoption

\section{Background}

To address persistent gaps in the use of evidence-based practices (EBPs), growing research has focused on the identification of specific implementation strategies, defined as methods or techniques used to enhance the adoption, implementation, and sustainment of a clinical program or practice $[1,2]$. The current paper describes findings from an initial test of a theory-driven implementation strategy designed to target malleable individuallevel determinants of behavior change (e.g., intentions to implement) among mental health clinicians in the education sector.

\section{Implementation strategies, determinants, and mechanisms}

Implementation strategies may be discrete, involving one specific process or action; multifaceted, including a combination of two or more discrete strategies; or blended, which are multifaceted strategies that have been explicitly protocolized or branded [3]. To be optimally effective, strategies should be selected and applied based on the specific multilevel determinants (i.e., barriers and facilitators [4]) they are intended to target $[1,5]$. Although the importance of inner setting (i.e., organizational) determinants is well established [6, 7], there are individual-level barriers (e.g., beliefs and attitudes) that commonly impede implementation outcomes [8-10]. Some studies have found that individual factors (especially attitudes) may be significantly more predictive of EBP use than organizational factors [11]. Further, while some organizational strategies have yielded encouraging results $[12,13]$, they are often time consuming and expensive [14]. Because implementation rests on the motivation, decisions, and behavior change of individuals within systems [15], it is critical to develop pragmatic (i.e., lowresource and contextually-appropriate) individual-level implementation strategies to target these determinants of implementation outcomes.

An important step of implementation strategy development is articulating their theorized mechanisms of change [5], although these are rarely evaluated [16, 17]. Theory is currently the best pathway through which to identify implementation mechanisms [5]. At the individual level, the theory of planned behavior (TPB) [18-20] has increasingly been applied to implementation behaviors $[21,22]$. The central tenet of TPB is that one of the best predictors of behavior is a person's behavioral intentions $[18,20]$, defined as motivations or conscious plans to exhibit particular behaviors. Behavioral intentions, in turn, are a function of an individual's attitudes (cognitive appraisals of the behavior in question), subjective norms (an individual's own estimate of the social pressure to perform the behavior), and perceived behavioral control (the extent to which an individual feels confident about being able to perform the behavior).

A recent meta-analysis of the TPB yielded an average effect size of .50 across a variety of patient behaviors (e.g., adherence to medical regimens) [23, 24]. The TPB is also the most commonly used social-cognitive theory for designing and evaluating the impact of implementation strategies [22]. The results of an implementationoriented systematic review [25] suggest that the variance in clinician behavior explained by intentions is similar to that reported among patients, although very few studies have evaluated TPB constructs as mechanisms of behavior change.

\section{Beliefs and attitudes for successful implementation in schools}

Grounded in the TPB, Beliefs and Attitudes for Successful Implementation in Schools (BASIS) is a blended implementation strategy developed to target individuallevel determinants of behavior change among schoolbased mental health clinicians. One in five youth experience a mental health problem [26] and $70-80 \%$ of youth mental health services are provided in schools [27-30]. Nevertheless, school mental health services are frequently not based on evidence for effectiveness [31-34]. Recent evidence shows that school mental health professionals' intentions to use EBP are strongly associated with subsequent use [35] and that implementation may be driven as much or more by individual-level determinants (e.g., attitudes) than by organizational processes [11]. BASIS employs strategies targeting each TPB component: (1) strategic education about EBP and intervention fidelity to improve attitudes toward EBP, (2) social influence techniques to alter perceptions of subjective norms, and (3) motivational interviewing techniques to enhance perceived behavioral control. Figure 1 displays the core BASIS components, as well as their respective mechanisms of 


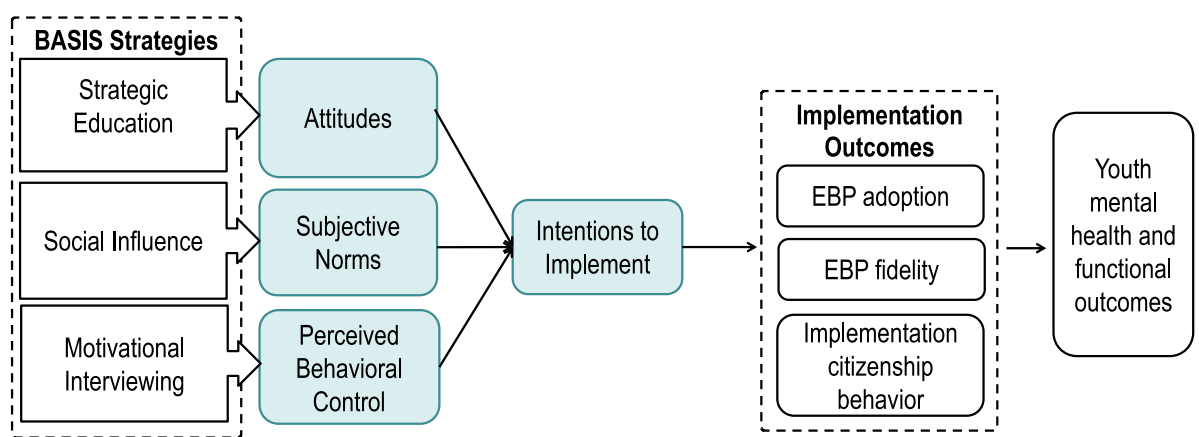

Fig. 1 BASIS components aligned with TPB hypothesized mechanisms of change and implementation outcomes. Colored boxes reflect the theory of planned behavior components

change (described in detail in "Methods" section). In practice, BASIS is a relatively brief $(3-4 \mathrm{~h})$ interactive session delivered to providers as an adjunct to training and consultation. Although most strategies tend to focus on the active implementation phase [36], BASIS is conceptualized as a pre-implementation strategy, delivered during the preparation phase, prior to and immediately after training and before consultation [6]. BASIS is not intended to replace other organizational implementation supports, such as coaching, high-quality training, and leadership. Rather it is designed to be compatible with and facilitative of other organizational (e.g., improving leadership) and innovation-specific (e.g., ongoing professional development connected to professional learning communities) implementation supports.

\section{Evaluations of previous versions of BASIS}

When delivered alongside training in a universal mental health EBP, a preliminary version of BASIS was associated with significantly more favorable attitudes toward EBP among 1181 teachers and administrators in a prepost trial $(d=1.03$; [37]). Attitudes, in turn, were associated with two measures of intervention integrity $(d=.51$ and $d=.67)$. Nevertheless, the preliminary version was relatively long and designed to target educator (rather than clinician) delivery of EBP. To address these limitations, our research team recently adapted BASIS for school mental health clinicians and found that it was perceived to be feasible, appropriate, and likely to have an impact on proximal mechanisms of behavior change [38].

\section{Study aims}

The current paper reports on a National Institute of Mental Health-funded study designed to conduct an initial randomized trial of the revised BASIS implementation strategy with a sample of school mental health clinicians, to augment training and consultation in a leading evidence-based trauma intervention (Cognitive
Behavioral Intervention for Trauma in Schools; CBITS) [39]. Although trauma is a growing concern among many educators and parents [40, 41], evidence-based interventions for youth exposed to trauma are rarely available [41]. High levels of clinician drop-out from, or discontinuation of, CBITS implementation efforts are a significant problem [42]. Evidence suggests that only $31 \%$ of sites follow through with delivery of group sessions after receiving training and consultative support. In the current study, we hypothesized that clinicians randomized to BASIS would demonstrate greater changes in target TPB mechanisms (i.e., attitudes, social norms, perceived behavioral control) from pre-training to post-training (hypothesis 1), would demonstrate higher intentions to implement (hypothesis 2), and would demonstrate higher rates of CBITS adoption and sustained participation in consultative implementation support activities (hypothesis 3). We also had an exploratory research question surrounding the extent to which changes in mechanisms would sustain from posttraining to end of year follow-up (research question 1). Finally, because our scientific questions related to individual-level determinants and processes, we did not include organizational implementation supports beyond training and consultation. This was intentional to isolate the influence of BASIS on hypothesized individual-level mechanisms of action and facilitate a nuanced understanding of how they function to support implementation. However, we evaluated aspects of implementation climate-an organizational-level variable-as an additional check on the comparability of the BASIS and control groups to rule out alternative explanations for any group differences.

\section{Methods}

To address the study aims, we conducted a pilot randomized trial (randomization at the clinician level) of the impact BASIS, relative to an attention control, on CBITS adoption. 


\section{Participants}

Mental health clinicians from two school districts in the Pacific Northwest participated. These two districts were selected based on their interest in integrating traumainformed services into secondary schools. Out of the 41 providers who were invited, 35 agreed to participate and were randomized. Following randomization, ten participants elected not to participate, leaving a final pool of 25 school mental health providers (12 in the BASIS condition, 13 in the control) attending the trainings (see CONSORT diagram; Fig. 2). This sample size was determined to be adequate for a preliminary trial, based on effect sizes documented in previous work [37] and the constraints of the funding mechanism. Table 1 displays participant demographics for the overall sample and

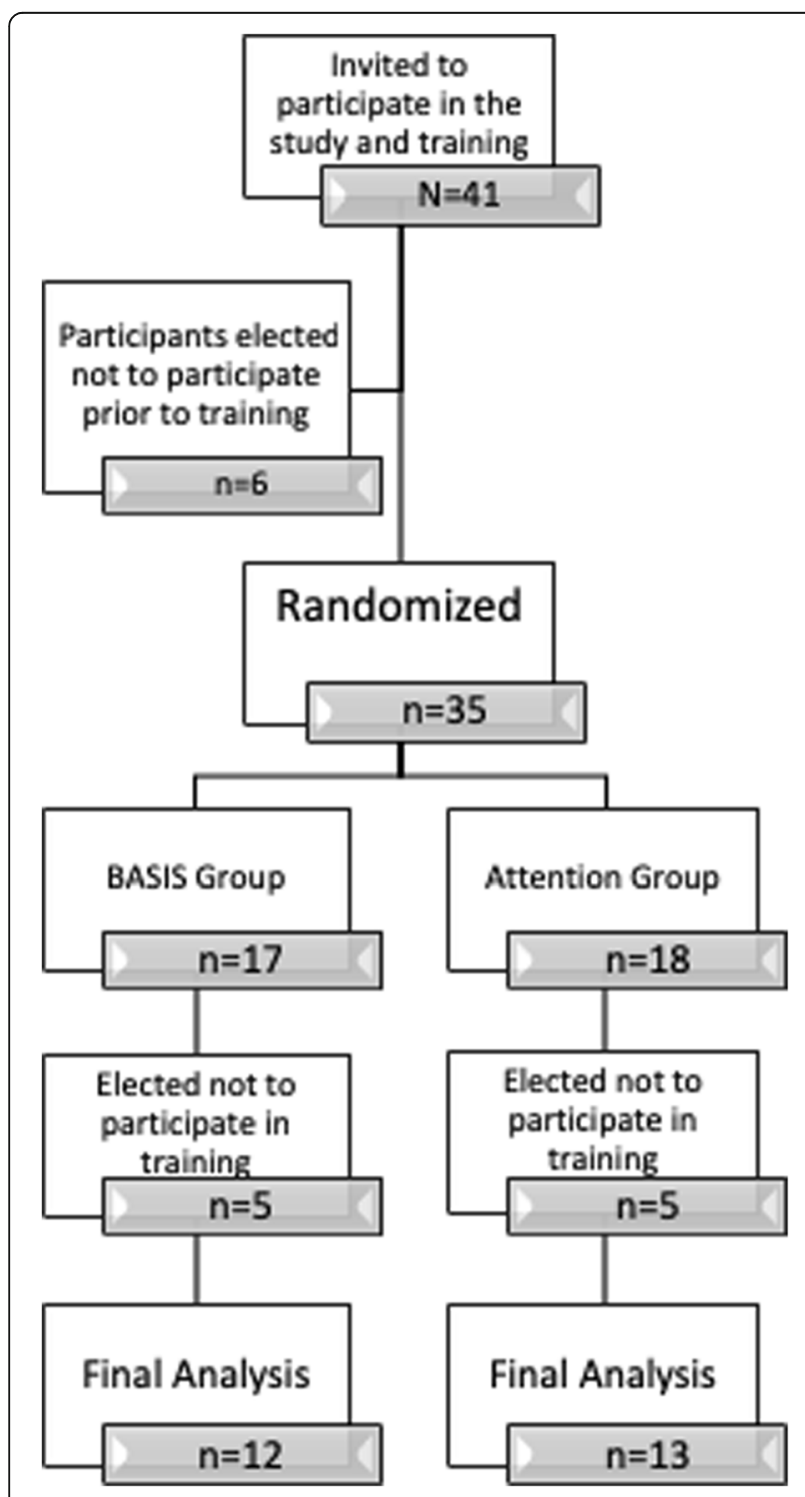

Fig. 2 CONSORT diagram for study participation stratified by condition, with $\chi^{2}$ analyses to test for condition differences.

\section{Procedures}

Recruitment flyers were sent via email to all eligible school mental health staff (i.e., those with credentials to provide mental health services) from the participating districts. Interested clinicians were contacted via phone, consented, and emailed a link to the pre-training survey using the Qualtrics online platform. Based on the pretraining survey, participants were assigned to condition via a nearest neighbor analysis using variables for grade level, EBP attitudes, social norms, perceived behavioral control, and intentions to implement EBPs, (see "Measures" section below) to identify matched pairs. Pairs were identified via the best match as identified by nearest Euclidean metrics, with a fixed $k$ of 2 ; in situations where three or more cases had competing best distances, the match was randomly selected.

Online pre- and post-training surveys were collected from both the attention control (AC) plus CBITS and the BASIS plus CBITS conditions. The CBITS training was then followed by biweekly consultation for 4 months with clinician participation tracked. To prevent condition contamination, training and consultation was provided separately (but using the same trainers) for the two conditions. After the consultation period, all providers completed online post-intervention measures.

\section{Study conditions \\ Attention control}

Providers randomly assigned to AC received a 3-hour $\mathrm{AC}$ intervention prior to CBITS training which was designed to control for dose, information provided, and interventionist effects. The AC facilitator defined, described, and advocated for EBP implementation in schools. Content was didactic, as is typical in trainings for school mental health providers.

\section{Basis}

The BASIS condition consisted of pre- and post-training sessions that bookended the CBITS training (see below). In this condition, clinicians received the three-hour group-based, interactive BASIS strategy, delivered by the same research team member who delivered the AC training to control for facilitator effects.

\section{BASIS implementation strategy}

Throughout BASIS, three components are embedded into the didactic sections and interactive activities, designed to target the three elements of TPB (see Table 2). These components are described below in accordance with guidelines for implementation strategy reporting [2]. 
Table 1 Demographic comparisons of the two groups

\begin{tabular}{|c|c|c|c|c|c|c|c|c|}
\hline & \multicolumn{2}{|c|}{ Total } & \multicolumn{2}{|c|}{ BASIS group $N=12$} & \multicolumn{2}{|c|}{ Control group $N=13$} & \multirow[t]{2}{*}{$x^{2}$} & \multirow[t]{2}{*}{$p$} \\
\hline & $N$ & $\%$ & $N$ & $\%$ & $N$ & $\%$ & & \\
\hline \multicolumn{9}{|l|}{ Gender } \\
\hline Female & 20 & $80.0 \%$ & 10 & $83.3 \%$ & 10 & $76.9 \%$ & \multirow[t]{2}{*}{0.031} & \multirow[t]{2}{*}{0.859} \\
\hline Male & 5 & $20.0 \%$ & 2 & $16.7 \%$ & 3 & $23.1 \%$ & & \\
\hline \multicolumn{9}{|l|}{ Race } \\
\hline Asian & 1 & $4.0 \%$ & 1 & $8.3 \%$ & 0 & $0.0 \%$ & \multirow[t]{6}{*}{2.72} & \multirow[t]{6}{*}{0.605} \\
\hline Black & 5 & $20.0 \%$ & 2 & $16.7 \%$ & 3 & $23.1 \%$ & & \\
\hline Hispanic & 1 & $4.0 \%$ & 0 & $0.0 \%$ & 1 & $7.7 \%$ & & \\
\hline Native & 0 & $0.0 \%$ & 0 & $0.0 \%$ & 0 & $0.0 \%$ & & \\
\hline White & 16 & $64.0 \%$ & 8 & $66.7 \%$ & 8 & $61.5 \%$ & & \\
\hline Multi-racial & 2 & $8.0 \%$ & 1 & $8.3 \%$ & 1 & $7.7 \%$ & & \\
\hline \multicolumn{9}{|l|}{ Age } \\
\hline 18 to 24 years old & 1 & $4.0 \%$ & 0 & $0.0 \%$ & 1 & $7.7 \%$ & \multirow[t]{6}{*}{2.62} & \multirow[t]{6}{*}{0.624} \\
\hline 25 to 34 years old & 5 & $20.0 \%$ & 2 & $16.7 \%$ & 3 & $23.1 \%$ & & \\
\hline 35 to 44 years old & 7 & $28.0 \%$ & 4 & $33.3 \%$ & 3 & $23.1 \%$ & & \\
\hline 45 to 54 years old & 7 & $28.0 \%$ & 2 & $16.7 \%$ & 5 & $38.5 \%$ & & \\
\hline 55 to 64 years old & 3 & $12.0 \%$ & 2 & $16.7 \%$ & 1 & $7.7 \%$ & & \\
\hline Missing & 2 & $8.0 \%$ & 2 & $16.7 \%$ & 0 & $0.0 \%$ & & \\
\hline \multicolumn{9}{|l|}{ Grade-level } \\
\hline Middle school & 13 & $52.0 \%$ & 6 & $50.0 \%$ & 7 & $53.8 \%$ & \multirow[t]{2}{*}{0.04} & \multirow[t]{2}{*}{0.582} \\
\hline High school & 12 & $48.0 \%$ & 6 & $50.0 \%$ & 6 & $46.2 \%$ & & \\
\hline
\end{tabular}

BASIS component 1: strategic education about EBP and intervention fidelity to improve attitudes toward EBP

BASIS incorporates strategic education to (1) help clinicians learn about the benefits of EBP for them professionally and for closing gap in access to highquality services for the youth they serve, as well as (2) alter any previously held beliefs they may have had about negative outcomes associated with EBP [43]. For example, clinicians estimate the potential access gap in their home schools and learn how delivery of EBPs is integral to addressing that gap. In addition, definitions and dimensions (e.g., adherence and dosage) of fidelity are presented, and clinicians are prompted to reflect on the critical importance of fidelity across a range of professions (e.g., engineering, farming, aviation). Moreover, clinicians learn about the outcomes of popular but ineffective practices (e.g., bloodletting, fat free diets) and how to recognize cognitive "shortcuts" that enhance individual vulnerability to adopting non-EBPs.

Table 2 BASIS components and content

\begin{tabular}{|c|c|}
\hline Component & Content \\
\hline Strategic education (attitudes) & $\begin{array}{l}\text { 1. Connecting EBP to student success } \\
\text { 2. Recognizing vulnerabilities to adopt non-EBPS } \\
\text { 3. Address common myths about EBP } \\
\text { 4. Evaluating evidence for practices } \\
\text { 5. Promoting understanding of fidelity of EBP }\end{array}$ \\
\hline Social influence (social norms) & $\begin{array}{l}\text { 1. Providing normative information } \\
\text { 2. Testimonials from experts } \\
\text { 3. Testimonials for similar others } \\
\text { 4. Evoking public commitments }\end{array}$ \\
\hline Motivational intervention (perceived behavioral control) & $\begin{array}{l}\text { 1. Professional values clarification } \\
\text { 2. Pros and cons activity to elicit change talk } \\
\text { 3. Anticipate implementation barriers } \\
\text { 4. Values-directed implementation goals } \\
\text { 5. "Ruler questions" (e.g., how confident?) }\end{array}$ \\
\hline
\end{tabular}




\section{BASIS component 2: social influence techniques to alter perceptions of subjective norms}

Recognizing that education alone is unlikely to change professional behavior, BASIS also relies on evidencebased social influence techniques. In particular, two strategies are used: (1) social proofing messages ("social proofs") that use data or testimonials to describe the behavior or attitudes of others, and (2) strategies to induce cognitive dissonance. Social proofs have been effectively used to reduce problem behaviors including alcohol use [44], illegal drug use [45], cigarette smoking [46], and eating disordered behaviors [47]. Evidence suggests that social proofs are most influential when people are given information about the current behavior of individuals with whom they closely identify. In BASIS, normative data and testimonials are used to validate clinician experiences of EBP implementation barriers (e.g., lack of time, low administrative support), and model commitments to problem-solving these barriers. In addition, expert testimonials address common myths about EBPs (e.g., that they are inflexible).

Strategies to induce cognitive dissonance operate on the premise that individuals strive for consistency between their attitudes and actions. Thus, desired behaviors can be increased by evoking commitments that are active (rather than passive), public (rather than private), and voluntary (rather than coerced) [48, 49]. In BASIS, clinicians set public goals for the upcoming training and for EBP implementation, and collaboratively generate potential solutions to overcome common barriers to implementation (e.g., time; lack of supervisor support). Clinicians' ideas are compiled and they are told their ideas will be shared with other clinicians who may encounter similar barriers. This activity is intended to position clinicians to freely and publicly advocate for potential solutions to EBP implementation.

\section{BASIS component 3: motivational interviewing techniques to enhance perceived behavioral control}

Motivational interviewing (MI) is a nondirective, personcentered counseling style for helping patients to explore and resolve ambivalence [50]. Decades of research have demonstrated significant effects of MI on adult and youth health behaviors [51-54] and on EBP implementation among teachers and primary care providers $[55,56]$.

The BASIS facilitator utilizes group MI techniques by adopting an empathic, supportive, and nondirective style to elicit self-motivational statements, encourage the elaboration of change talk, and enhance perceived behavioral control. For example, clinicians engage in an evidence-based values affirmation activity, which has been shown to decrease defensiveness toward change and enhance motivation to engage in value-congruent behavior [57]. Clinicians are asked to reflect on and share with the group reasons why they chose their profession and why they continue despite challenges. Clinicians also anticipate barriers that may arise in implementation, engage in collaborative problem-solving to brainstorm ways to address those barriers, answer standard MI "ruler" questions (e.g., "On a scale of 1-10, how confident are you in your ability to implement an EBP?") [50], and engage in group discussion about what is needed to increase their ratings. Additionally, providers are asked to recall other times when they have successfully made changes in their careers, highlighting their capability to take on and implement innovative practices. Throughout the session, the facilitator uses standard MI techniques: elaborate on change talk, express empathy, roll with resistance, and emphasize autonomy.

\section{CBITS training and consultation}

CBITS is an evidence-based intervention consisting of ten group sessions, 1-3 individual sessions, two parent psychoeducational sessions, and one teacher educational session. Used with students in grades 5-12 who have witnessed or experienced traumatic life events, CBITS has been shown to reduce symptoms of trauma and depression and improve academic outcomes [58, 59]. Candidate students are eligible for CBITS based on screening procedures to identify trauma-exposed students from the entire population of students in a school who are in need of and would benefit from CBITS. CBITS was developed to be used by school mental health clinicians, has established training protocols, and provides structured post-training consultation supports [60, 61].

After receiving BASIS or AC, all providers participated in a standard, 1.5-day CBITS training delivered by the same certified trainers, blinded to condition. CBITS training included best practices for educational meetings: didactic content delivery, rehearsal activities, and performancebased feedback [62]. A trained CBITS consultant, also blind to condition, provided biweekly post-training group consultation to providers who received either BASIS and AC. Participation in post-training group consultation was an expectation of clinician enrollment in the study. Consultation groups (6-8 providers/each) were formed within BASIS or AC conditions to avoid contamination.

\section{Measures}

A detailed description of all study measures, including reliabilities in the current sample, is provided in Additional file 1.

\section{Attitudes}

The school version of the Evidence-Based Practice Attitudes Scale (EBPAS) [63] is a 26-item adaptation of the original EBPAS [64]. Subscales include Requirements, Appeal, 
Openness, Divergence, Fit, and Burden. Attitudes were measured at baseline, post, and follow-up time points.

\section{Perceived social norms}

The modified subjective norms measure, based on guidelines for developing reliable and valid measures of theory of planned behavior (TPB) constructs [65, 66], captures two types of EBP implementation-related subjective norms: injunctive (what a social group would approve of) and descriptive (how a social group actually behaves). Perceived social norms were measured at baseline, post, and follow-up time points.

\section{Perceived behavioral control}

A modified version of the Teacher Self-Efficacy Scale [67] with ten items was used to assess perceived behavioral control in implementing EBP. Perceived behavioral control was measured at baseline, post, and follow-up time points.

\section{Implementation citizenship}

The School Implementation Citizenship Behavior Scale (S-ICBS) [68], modified from the original Implementation Citizenship Behavior Scale [69], measures clinicians' perceptions regarding how school staff engage with EBPs within their specific school context. Subscales include Helping others, Keeping informed, Taking Initiative, and Advocacy. Implementation citizenship was measured at baseline, post, and follow-up time points.

\section{Behavioral intentions to implement}

The Modified Intentions to Use Scale [70] assesses school mental health providers' intentions to implement EBP. This scale was developed based on established guidelines for developing behavioral intention measures using the TPB $[65,66]$ and was administered pre and post BASIS and $\mathrm{AC}$ conditions to examine changes in school mental health providers' intentions to implement CBITS.

\section{BASIS fidelity}

An observational fidelity tool was developed by the research team to capture adherence to 33 total components of BASIS. Two coders independently rated a video recording of the BASIS condition; one rater coded 97\% and the second rater coded $94 \%$ of 33 BASIS components as having been delivered. The raters failed to jointly classify any component as not delivered. In situations such as this, Cohen's kappa statistic is an inappropriate measure of interrater reliability [72]. Therefore, we report that raters were in agreement on $91 \%$ of the components delivered in the BASIS condition, and that a minimum of $91 \%$ of components were delivered during BASIS. Only one rater coded the AC condition; it covered $21 \%$ of BASIS components.

\section{Consultation engagement}

Each participant was provided the opportunity to attend up to 13 consultation phone calls with a CBITS consultant. At the end of each call, the consultant recorded who attended the consultation call, whether or not participants completed homework (when applicable), and overall engagement in the call. Ongoing participation in consultation included (1) attending at least one posttraining consultation session, (2) number (\%) of consultation sessions attended, and (3) days post-training to consultation dropout.

\section{Adoption}

Adoption was operationalized as the initiation of a CBITS group at any point during study participation, the number of days until the first CBITS session, and the number of days until implementation dropout. Previous research indicates that the vast majority of CBITS groups, once initiated, are completed [42].

\section{Implementation climate}

The school-adapted Implementation Climate Scale [68], modified from the original Implementation Climate Scale [71], measures clinician perceptions of the extent to which EBP implementation is expected, supported, and rewarded in their setting. Subscales include Focus on EBP, Educational Support for EBP, Recognition for EBP, Rewards for EBP, Use of Data to support EBP, Use of Existing Supports/Infrastructure for EBP Implementation, and Degree of EBP Integration. Because it was not theorized to change as a result of BASIS, implementation climate was measured at post-BASIS and follow-up time points. While implementation climate as a construct exists at the organization level, the construct reflects individuals' reactions to the context of a particular organization and, therefore, also may be measured at the individual level.

\section{Data analytic approach}

Means and standard deviations were calculated for all scales and subscales at baseline, post-training, and follow-up. For all variables collected at baseline and post-training, we computed a series of ordinary least square (OLS) regression models predicting post-training score, using baseline score as covariate and condition as a predictor. Similarly, for all variables collected at posttraining and follow-up, we computed a series of OLS regression models predicting follow-up score, using post-training score as a covariate and condition as a predictor. Some variables were not collected at baseline; for these, we computed independent sample $t$ tests to compare conditions at post-training. There was very little missing data: one person in the BASIS group skipped a 
section of the baseline survey, and therefore was missing data on the EBPAS, social norms, self-efficacy, and EBP intentions. No other participants were missing data. We imputed missing values for these variables using OLS regression models using all available data as predictors. We chose OLS regression rather than multiple imputation or maximum likelihood methods due to the small sample size, small amount of missing data, and relative ease of these calculations. Due to small sample size and limited statistical power, we present standardized coefficients $(\beta \mathrm{s})$ and 95\% confidence intervals (significant effects at $p<.05$ are highlighted to be consistent with convention), and we do not adjust for familywise error rate. Because these are standardized coefficients, they can be interpreted as effect sizes and compared across variables for strength of effect, which we interpret using .1 as a small effect (a tenth of a standard deviation), .3 as a medium effect (a third of a standard deviation), and .5 as a large effect (half of a standard deviation). Tables provide all results; in the section below, we report on all effects of $\beta=.3$ or larger.

\section{Results}

Hypothesis 1: pre- to post-training change in mechanisms Table 3 depicts the unadjusted mean scores and standard deviations for all outcome measures at baseline, post-training, and follow-up, as well as standardized coefficients for intervention effects obtained via multiple regressions using baseline scores as covariates.

All outcomes for pre- to post-training that had medium or greater effect sizes were in the predicted direction. The attention control group did not improve from pre- to post-training on any variables, as indicated by raw score. Self-efficacy had a medium effect size with the BASIS group remaining stable while the AC deteriorated $(\beta=.36, C I=-.02$ to .74$)$. Three of the six EBP attitudes subscales had a medium effect size, with the BASIS group showing an improving trend and the $\mathrm{AC}$ group showing deterioration for the subscales of Appeal $(\beta=.489, \mathrm{CI}=.159$ to .820$)$, Openness $(\beta=.49, \mathrm{CI}=.17$ to .80$)$, and Fit $(\beta=.47, C I=.18$ to .77$)$. Both of the Norms subscales had a medium effect size, with the BASIS group showing an improving trend and the AC group showing deterioration for Descriptive $(\beta=.47, \mathrm{CI}$ $=.86$ to .09$)$ and Injunctive Norms $(\beta=.31, \mathrm{CI}=.72$ to -.10). None of the Implementation Citizenship Behaviors subscales had a medium or greater effect size.

\section{Hypothesis 2: intentions to implement}

Intentions to implement showed a medium effect size, with the BASIS group remaining stable while the AC group deteriorated ( $\beta=.34, \mathrm{CI}=.01$ to .67 ).

\section{Hypothesis 3: consultation participation, engagement, and CBITS adoption}

Eight out of 12 (66.7\%) BASIS clinicians participated in at least one post-training CBITS consultation session, as compared to six out of 13 (46.2\%) for AC; this difference was not statistically significant $\left(\chi^{2}=1.07, p=.30\right)$. We restricted the following three analyses to only include those 14 individuals who attended at least one consultation session. The BASIS group attended a mean of 4.1 sessions and the AC attended a mean of 5.8 , which was not statistically significant $\left(t_{(12)}=.78, p=.45\right)$. The average engagement score for BASIS was $1.7(S D=16)$ and for AC was $2.5(S D=1.9)$, also not statistically significant $\left(t_{(12)}=.91, p=.38\right)$. The average percentage of CBITS consultation homework assignments completed, after prorating for number of sessions attended, for BASIS was $27 \%(S D=36 \%)$ and for $\mathrm{AC}$ was $43 \%(S D=30 \%)$, also not statistically significant $\left(t_{(12)}=.89, p=.39\right)$.

There were 15.4\% (two of 13) attention control participants and $25.0 \%$ (3 of 12) BASIS participants who began a CBITS group during the study; this difference was not statistically significant $\left(\mathrm{x}^{2}=.36, p=.55\right)$. A Kaplan-Meier time-to-event analysis (see Fig. 3) found that the median dropout from their commitment to implement a CBITS group was 32 days for the AC group, and 134 days for the BASIS group, which was large but not a statistically significant difference (log-rank $\chi^{2}=1.08, p=.30$; Breslow $\chi^{2}=2.2, p=.14$; Tarone-Ware $\left.\chi^{2}=1.6, p=.21\right)$. There were no significant differences in the number of days until a CBITS group began (log-rank $\chi^{2}=.18, p=.67$; Breslow $X^{2}=.24, p=.63$; Tarone-Ware $\chi^{2}=.21, p=.65$ ), though medians could not be calculated because fewer than half of participants implemented CBITS (Fig. 4).

\section{Exploratory research question: post-training to end-of- year follow-up change in mechanisms}

For post-training to the follow-up timepoints (Table 3), there were fewer effects of medium or greater size. Two of the EBP Attitudes subscales had a medium effect size. The strongest effects from post-training to follow-up were for EBPAS Divergence $(\beta=.48, \mathrm{CI}=.26$ to .70$)$. For this variable, higher scores indicate that participants are more likely to report that research-based interventions are clinically useful or as important as clinical experience. EBPAS Requirement also had a medium effect $(\beta$ $=.40, \mathrm{CI}=-.01$ to .80$)$. For both EBPAS subscales, the BASIS group showed an improving trend while the AC group deteriorated. For Social Norms, both subscales had a medium effect size, including Injunctive $(\beta=-.36$, $\mathrm{CI}=.10$ to -.82$)$ and Descriptive Norms $(\beta=-.35, \mathrm{CI}=$ -10 to -.80 ). For Injunctive Norms, the BASIS group deteriorated while the AC group showed stronger norms. For Descriptive Norms, the BASIS group deteriorated while the AC group remained stable. There were 
Table 3 Mean scores, standard deviations, and BASIS impact for outcomes at each timepoint

\begin{tabular}{|c|c|c|c|c|c|c|c|c|c|c|c|c|}
\hline \multirow[t]{2}{*}{ Measures } & \multirow[t]{2}{*}{ Condition } & \multirow[t]{2}{*}{$n$} & \multicolumn{2}{|c|}{ Baseline } & \multicolumn{2}{|l|}{ Post } & \multirow{2}{*}{$\begin{array}{l}\text { Follow-up } \\
\text { Mean }\end{array}$} & \multicolumn{3}{|c|}{ Baseline to post regression } & \multicolumn{2}{|c|}{ Post to FU Regression } \\
\hline & & & Mean & SD & Mean & SD & & SD & Beta & $95 \% \mathrm{Cl}$ & Beta & $95 \% \mathrm{Cl}$ \\
\hline \multicolumn{13}{|l|}{ Attitudes toward EBPs (EBPAS) } \\
\hline \multirow[t]{2}{*}{ Requirement } & BASIS & 11 & 8.85 & 2.40 & 9.45 & 2.81 & 9.89 & 1.17 & .106 & $-.211, .423$ & .396 & $-.010, .802$ \\
\hline & Attention & 13 & 9.69 & 1.65 & 9.54 & 2.26 & 7.31 & 4.39 & & & & \\
\hline \multirow[t]{2}{*}{ Appeal } & BASIS & 11 & 12.74 & 3.35 & 14.18 & 1.78 & 13.33 & 2.18 & .489 & $.158, .820$ & -.026 & $-.379, .327$ \\
\hline & Attention & 13 & 13.54 & 1.94 & 12.08 & 2.72 & 12.00 & 3.06 & & & & \\
\hline \multirow[t]{2}{*}{ Openness } & BASIS & 11 & 11.76 & 2.20 & 13.73 & 1.74 & 12.22 & 1.72 & .488 & $.174, .802$ & -.121 & $-.405, .163$ \\
\hline & Attention & 13 & 13.00 & 2.48 & 12.00 & 2.83 & 11.62 & 3.15 & & & & \\
\hline \multirow[t]{2}{*}{ Divergence } & BASIS & 11 & 12.53 & 1.85 & 12.27 & 2.37 & 13.00 & 2.18 & -.105 & $-.517, .307$ & .481 & $.261, .701$ \\
\hline & Attention & 13 & 13.31 & 1.44 & 13.00 & 1.83 & 12.08 & 1.38 & & & & \\
\hline \multirow[t]{2}{*}{ Fit } & BASIS & 11 & 23.03 & 3.76 & 24.18 & 2.93 & 22.56 & 3.88 & .473 & $.180, .766$ & .191 & $-.271, .653$ \\
\hline & Attention & 13 & 22.31 & 3.84 & 19.77 & 4.51 & 19.31 & 3.99 & & & & \\
\hline \multirow[t]{2}{*}{ Burden } & BASIS & 11 & 10.70 & 2.45 & 10.18 & 3.46 & 9.11 & 3.95 & .277 & $-.143, .697$ & .074 & $-.361, .509$ \\
\hline & Attention & 13 & 11.08 & 2.25 & 8.62 & 3.97 & 7.92 & 3.48 & & & & \\
\hline \multicolumn{13}{|l|}{ Social norms } \\
\hline \multirow[t]{2}{*}{ Injunctive norms } & BASIS & 11 & 0.90 & 1.14 & 1.36 & 1.04 & 0.81 & 1.48 & .309 & $.721,-.103$ & .357 & $.102,-.817$ \\
\hline & Attention & 13 & 1.00 & 0.82 & 0.81 & 0.75 & 1.42 & 0.62 & & & & \\
\hline \multirow[t]{2}{*}{ Descriptive norms } & BASIS & 11 & 1.11 & 1.26 & 1.45 & 0.76 & 0.64 & 0.90 & .474 & $.859, .089$ & .348 & $.102,-.798$ \\
\hline & Attention & 13 & 1.21 & 0.89 & 0.60 & 1.04 & 0.69 & 0.90 & & & & \\
\hline \multirow[t]{2}{*}{ Self-efficacy } & BASIS & 9 & 35.11 & 6.07 & 35.44 & 4.28 & 31.78 & 5.45 & .360 & $-.020, .740$ & -.297 & $-.667, .073$ \\
\hline & Attention & 13 & 34.85 & 4.76 & 31.08 & 5.81 & 32.08 & 5.62 & & & & \\
\hline \multicolumn{13}{|c|}{ Implementation citizenship behaviors (ICBS) } \\
\hline \multirow[t]{2}{*}{ Helping others } & BASIS & 11 & 2.73 & 0.65 & 2.55 & 1.11 & 2.15 & 1.26 & .166 & $-.185, .517$ & -.082 & $-.424, .260$ \\
\hline & Attention & 13 & 1.95 & 0.97 & 1.85 & 0.95 & 1.82 & 1.21 & & & & \\
\hline \multirow[t]{2}{*}{ Keeping informed } & BASIS & 11 & 2.85 & 0.70 & 2.91 & 0.67 & 2.41 & 0.98 & .102 & $-.163, .366$ & -.275 & $-.569, .020$ \\
\hline & Attention & 13 & 2.51 & 1.18 & 2.49 & 1.22 & 2.64 & 1.13 & & & & \\
\hline \multirow[t]{2}{*}{ Taking initiative } & BASIS & 11 & 3.11 & 0.65 & 2.86 & 0.81 & 2.44 & 1.16 & .087 & $-.254, .428$ & -.271 & $-.493,-.050$ \\
\hline & Attention & 13 & 2.60 & 0.90 & 2.48 & 0.89 & 2.58 & 0.98 & & & & \\
\hline \multirow[t]{2}{*}{ Advocacy } & BASIS & 11 & 3.36 & 0.54 & 3.15 & 0.84 & 2.69 & 1.24 & -.053 & $-.434, .327$ & -.218 & $-.439, .003$ \\
\hline & Attention & 13 & 2.75 & 0.82 & 2.77 & 0.79 & 2.78 & 0.97 & & & & \\
\hline \multirow[t]{2}{*}{ Mean total } & BASIS & 11 & 3.01 & 0.51 & 2.87 & 0.79 & 2.42 & 1.09 & .066 & $-.244, .376$ & -.252 & $-.489,-.016$ \\
\hline & Attention & 13 & 2.45 & 0.87 & 2.40 & 0.85 & 2.46 & 0.98 & & & & \\
\hline Intentions to implement CBITS & BASIS & 9 & 31.00 & 5.68 & 30.89 & 4.91 & 24.78 & 7.17 & .339 & $.007, .671$ & -.154 & $-.597, .289$ \\
\hline & Attention & 13 & 29.54 & 4.82 & 24.08 & 8.37 & 24.08 & 7.92 & & & & \\
\hline
\end{tabular}

Bolded betas represent significant effects. Regression models examine impact of condition, controlling for baseline score

no other medium or greater effect sizes for any construct, including self-efficacy, implementation citizenship, or intentions to implement CBITS. However, one implementation citizenship subscale was statistically significant, Taking Initiative $(\beta=-.27, \mathrm{CI}=-.49$ to -.05$)$, and the total score for implementation citizenship was statistically significant $(\beta=-.25, \mathrm{CI}=-.49$ to -.02$)$. Finally, as can be seen in Table 4 , implementation climate was comparable across BASIS and AC groups at post, with no statistically significant differences between the groups at either timepoint, and no effect sizes greater than small effects.

\section{Discussion}

The current study evaluated the influence of BASIS-a blended, individual-level pre-implementation strategyon theorized mechanisms of behavior change, sustained participation in post-training implementation supports, and CBITS adoption. Findings suggested that, consistent with its underlying theory of change, BASIS had significant 


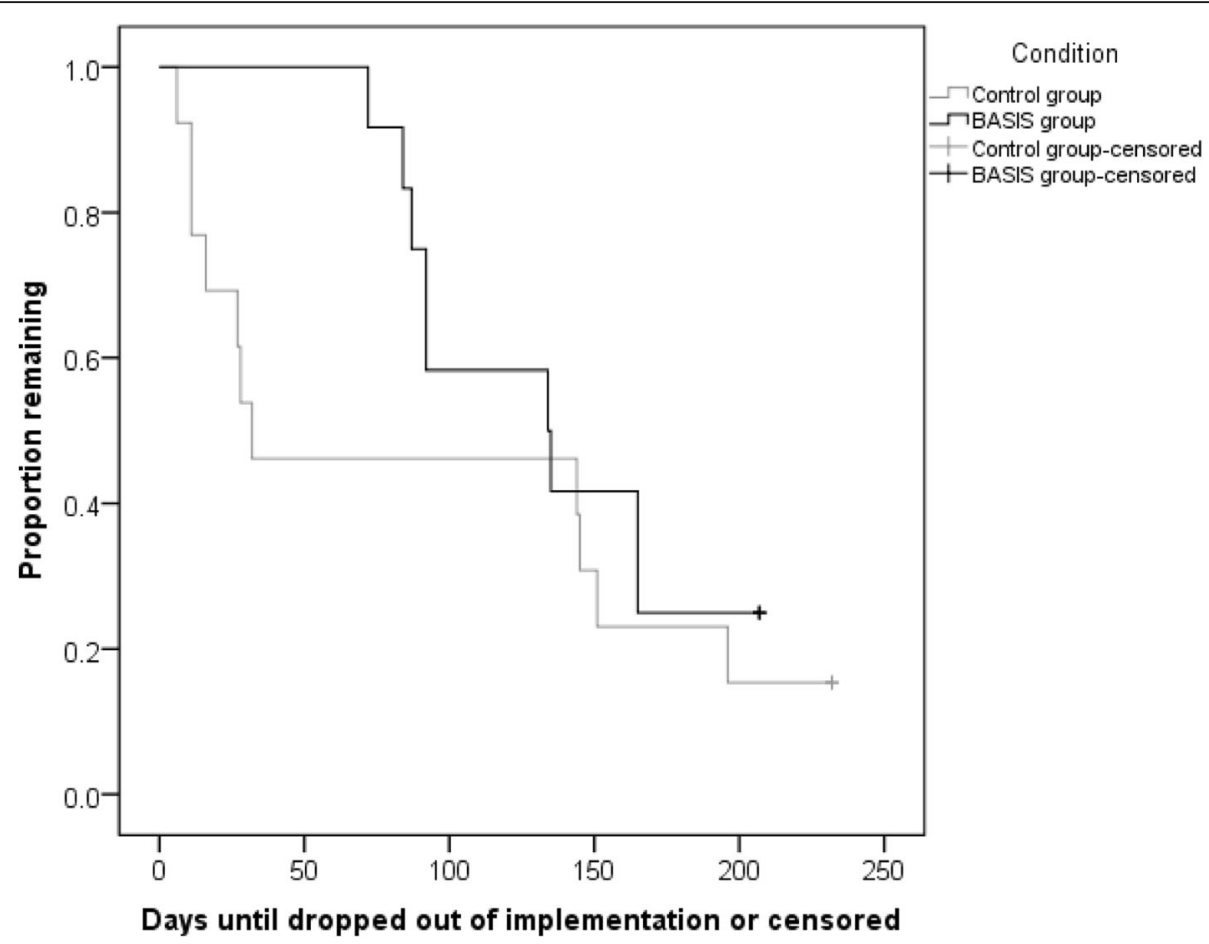

Fig. 3 Time-to-event analysis: days until providers dropped out of CBITS implementation

effects on the majority of its proximal outcomes, with BASIS clinicians demonstrating higher levels on target mechanisms relative to the attention control at posttraining. This included medium to large effect sizes for attitudes (appeal, openness, fit), descriptive social norms, self-efficacy, and intentions to implement CBITS. Relative to the attention control, clinicians in the BASIS condition persisted in consultation over four times longer than controls (i.e., 134 versus 32 days), but this difference was not statistically significant. Evaluation of the mechanisms at the follow-up time point suggested that many changes in mechanisms did not persist over the full 4-month period.

Table 4 Implementation climate means, standard deviations, difference across conditions, and BASIS impact from post to follow-up

\begin{tabular}{|c|c|c|c|c|c|c|c|c|c|c|}
\hline \multirow[t]{2}{*}{ Measure } & \multirow[t]{2}{*}{ Condition } & \multirow[t]{2}{*}{$n$} & \multicolumn{2}{|l|}{ Post } & \multicolumn{2}{|c|}{ Follow-up } & \multicolumn{2}{|c|}{ Post-training independent $T$ test } & \multicolumn{2}{|c|}{ Post to FU regression } \\
\hline & & & Mean & SD & Mean & SD & $t$ & $p$ & Beta & $95 \% \mathrm{Cl}$ \\
\hline \multirow[t]{2}{*}{ ICS focus } & BASIS & 11 & 2.67 & 0.87 & 2.33 & 1.02 & -.905 & .375 & -.065 & $-.522, .392$ \\
\hline & Attention & 13 & 2.33 & 0.92 & 2.33 & 0.77 & & & & \\
\hline \multirow[t]{2}{*}{ ICS education } & BASIS & 11 & 2.52 & 0.89 & 2.23 & 0.85 & -.961 & .347 & -.125 & $-.596, .346$ \\
\hline & Attention & 13 & 2.18 & 0.82 & 2.33 & 0.58 & & & & \\
\hline \multirow[t]{2}{*}{ ICS recognition } & BASIS & 11 & 2.73 & 0.61 & 2.10 & 0.74 & -1.136 & .268 & -.299 & $-.754, .156$ \\
\hline & Attention & 13 & 2.41 & 0.73 & 2.46 & 0.89 & & & & \\
\hline \multirow[t]{2}{*}{ ICS reward } & BASIS & 11 & 1.24 & 0.83 & 0.80 & 0.77 & -.907 & .374 & -.139 & $-.591, .313$ \\
\hline & Attention & 13 & 0.97 & 0.62 & 0.90 & 0.77 & & & & \\
\hline \multirow[t]{2}{*}{ ICS data } & BASIS & 11 & 2.36 & 0.74 & 1.58 & 0.76 & -1.080 & .292 & -.160 & $-.625, .305$ \\
\hline & Attention & 13 & 1.94 & 1.10 & 1.73 & 1.02 & & & & \\
\hline \multirow[t]{2}{*}{ ICS support } & BASIS & 11 & 2.21 & 1.00 & 1.70 & 1.01 & -.133 & .896 & -.067 & $-.532, .398$ \\
\hline & Attention & 13 & 2.15 & 1.13 & 1.82 & 1.07 & & & & \\
\hline \multirow[t]{2}{*}{ ICS integrate } & BASIS & 11 & 2.18 & 0.85 & 1.53 & 1.06 & -1.160 & .258 & -.141 & $-.600, .318$ \\
\hline & Attention & 13 & 1.77 & 0.88 & 1.62 & 0.80 & & & & \\
\hline \multirow[t]{2}{*}{ ICS total } & BASIS & 11 & 2.28 & 0.62 & 1.73 & 0.73 & -1.175 & .252 & -.149 & $-.635, .337$ \\
\hline & Attention & 13 & 1.96 & 0.71 & 1.87 & 0.61 & & & & \\
\hline
\end{tabular}




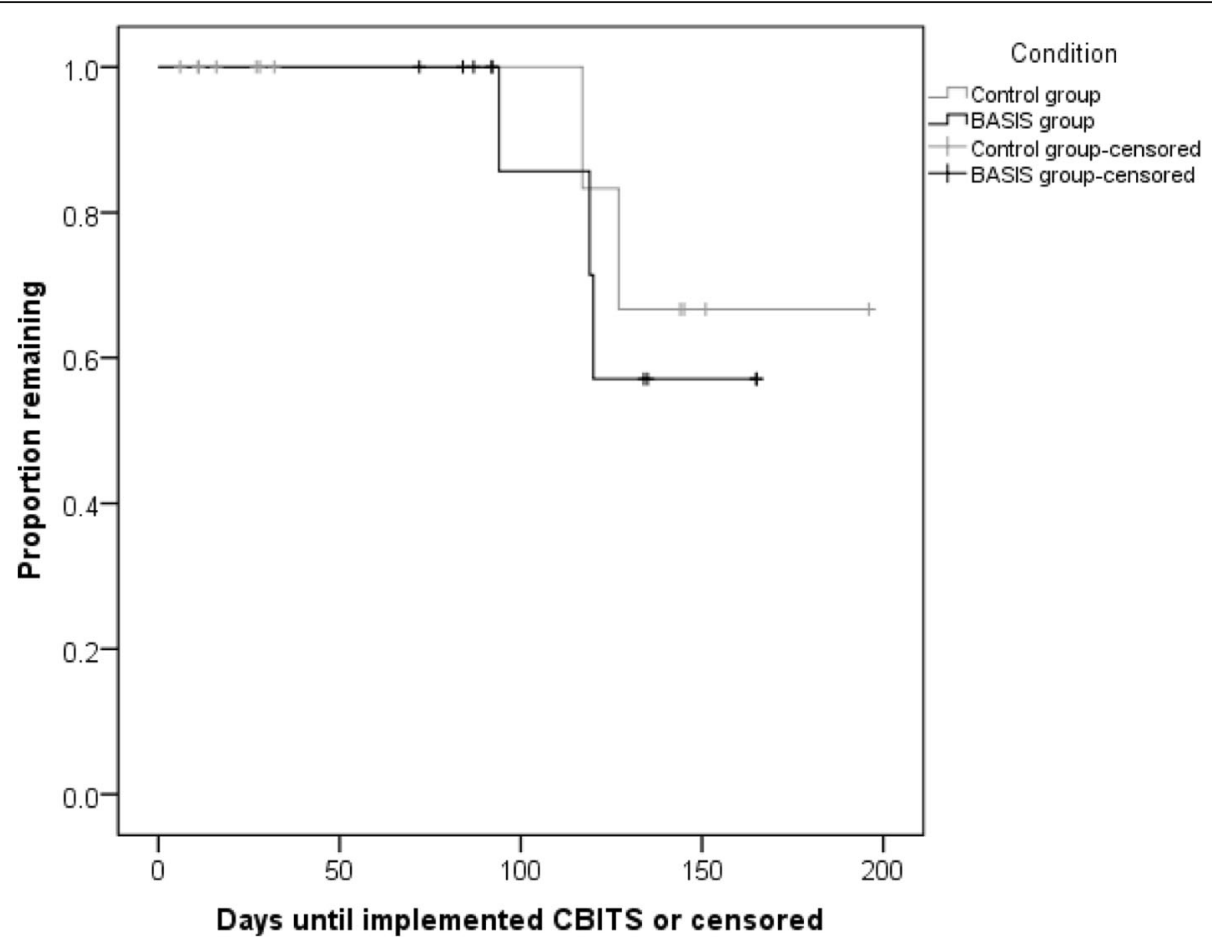

Fig. 4 Time-to-event analysis: days until providers initiated a CBITS group

Further, BASIS did not demonstrate detectable effects on clinician adoption behaviors among the small number of clinicians who delivered the CBITS intervention $(n=5$; three BASIS and two AC), indicating that post-training changes on the target individual-level determinants may be insufficient to influence implementation behaviors above and beyond training and consultation.

\section{BASIS and the theory of planned behavior}

The impact of BASIS on the TPB mechanisms-particularly attitudes (appeal, openness, divergence, fit) and descriptive norms-and intentions to implement is aligned with theory and prior research. Similar to the current study, prior interventions that aimed to impact healthcare providers' attitudes, subjective norms, perceived behavioral control, and intentions also found significant changes immediately post-training, but did not see sustained effects over time [73].

Unlike previous studies, however, we did not find evidence that these mechanisms facilitated delivery of CBITS. In a systematic review of the impact of social cognitive theories on healthcare professional's intentions and behaviors, Godin and colleagues [22] found that $35 \%$ of the variance in behaviors across studies was associated with elements of the TPB. In particular, of all of the TPB mechanisms, healthcare professionals' beliefs about their capabilities, including their perceived behavioral control (i.e., self-efficacy), was the most consistent predictor of behavior. In the current study, BASIS demonstrated a medium, but nonsignificant, effect on perceived behavioral control. It is possible that perceived behavioral control is an especially important component of the TPB that is essential to influence in order to facilitate behavior change and EBP implementation, but may be particularly challenging to impact in a time-limited pre-implementation intervention. Future implementation efforts may place more emphasis on enhancing this construct. In contrast, subjective norms are often considered the weakest or most inconsistent predictor of behavioral change [18, 74-76]. Although BASIS had moderate to strong impacts on both injunctive and descriptive norms at the post timepoint, this domain of the TPB may be necessary but insufficient to facilitate uptake and sustainment of an EBP. Furthermore, despite initial improvements, both types of social norms deteriorated for BASIS clinicians at the follow-up time point, reaching levels that were comparable to baseline or the control condition. As described below (see "Potential BASIS revisions" section), this suggests that BASIS, while time limited and efficient, might result in effects that are difficult to maintain without additional supports, such as booster sessions.

Although BASIS was explicitly designed to isolate and influence individual-level processes, a wealth of implementation literature indicates that aspects of the inner organizational setting-such as organizational culture, climate, and leadership-are critical to implementation success $[11,14]$. Although data collected in the current 
study demonstrated comparable implementation climate across the BASIS and control conditions, organizational issues may still have operated to decrease CBITS implementation in both groups. BASIS is not intended to be a stand-alone implementation strategy. Ultimately, we expect that combining individually oriented implementation strategies, such as BASIS, with organizational strategies (e.g., [13]) may have a synergistic effect on implementation and service outcomes.

It is also important to note that the majority of previous literature documenting significant effects of the TPB on clinician's behavior has been conducted in relation to physical healthcare as opposed to mental health service delivery. In these studies, the desired behavior changes (e.g., prescribing, referring patients, performing an examination, documentation) sometimes differ in complexity from the multifaceted psychosocial interventions that typify mental health EBPs [76]. It is possible that it is particularly challenging to apply the TPB to enact change in mental health clinicians' use of EBP, perhaps due to the complexity of the interventions. CBITS is a complex intervention with components that may limit its usability and ease of implementation. For instance, the first step to adopt CBITS involves administering screening procedures to detect youth who have experienced trauma and are in need of a trauma-informed intervention. Thus, initiating the delivery of CBITS involves significant motivation and effort on the part of the clinician to conduct trauma screening which involves sensitive questions and is not typical of routine practice in schools [58]. Additionally, because it is group-based, CBITS requires the coordination of many different student and staff calendars. Parent sessions, when conducted, add additional complexity.

\section{Applications of BASIS}

Although BASIS did not demonstrate a sustained impact on proximal mechanisms through a 4-month follow-up, there were detectable improvements immediately following delivery. This suggests that BASIS may be a helpful strategy for generating an initial spike of energy at the beginning of an implementation process, a critical period when the introduction of new knowledge and behavior expectations may result in a short-term decrease in skill level [77]. This may be particularly useful for low complexity interventions. Alternatively, BASIS may have enough of an impact on TPB mechanisms to affect behavior change within a setting that is particularly conducive to new implementation efforts (e.g., favorable implementation leadership, climate, and citizenship behavior $[69,71,78,79])$. In such settings, BASIS may be combined with-or integrated into-"cornerstone" implementation strategies such as training and posttraining consultation efforts [80] to enhance their effects.

\section{Potential BASIS revisions}

The findings suggest that, while the BASIS strategy was effective in shifting its proximal targets in the short term, additional supports may be necessary to ensure that gains persist over time and fully translate into changes in implementation behaviors. Previous research has demonstrated that change commitment fluctuates across the year, with overall decreasing trends [81], suggesting that behavioral intentions may change in response to a range of multilevel influences. BASIS was initially conceptualized as a pragmatic and time-limited implementation strategy, delivered immediately before and following training in an EBP. Revisions to the BASIS strategy that capitalize on its effectiveness in changing clinician perceptions and engagement in the short term, but increase its sustained impact, may include either (1) more explicit post-training barrier anticipation and action/coping planning and/or (2) incorporation of booster sessions to maintain shifts in attitudes, social norms, perceived behavioral control, and behavioral intentions.

\section{Action and coping planning}

Although BASIS buffered against decreases in implementation intentions following the training, these results highlight a potential need to develop BASIS components that more explicitly translate those intentions into implementation behaviors. The Health Adaption Process Approach (HAPA $[82,83]$ ) is a stage model (in contrast to the TPB, which is considered a continuum model) that outlines processes of behavior change with the aim of minimizing the intention-behavior gap. HAPA distinguishes between a motivational phase, during which individuals develop their intentions to act, and a volitional phase, where those intentions are supported and transformed into actions. Action and coping planning are essential components of the volitional phase. Through action planning, individuals (e.g., clinicians) outline a clear and detailed plan of when and how they would implement the intended practice (e.g., CBITS). Coping planning allows clinicians to identify potential barriers that they expect to face in implementing the practice, and determine strategies for overcoming those barriers. Action and coping planning have been associated with higher levels of behavioral change [84]. Incorporating action and coping planning into BASIS will likely enhance clinicians' abilities to set and pursue goals related to adoption and eventual high-fidelity delivery of CBITS.

\section{Boosters}

In addition, incorporating booster sessions to maintain positive shifts in proximal mechanisms may allow BASIS to facilitate adoption and delivery of the EBP over time once providers return to the realities of their jobs post- 
training. Boosters for specific EBPs have been shown to enhance adoption in school settings [85-88] and facilitate strong impacts on service recipient outcomes $[89,90]$. BASIS booster sessions may revisit core BASIS components (e.g., strategic education to enhance attitudes toward EBPs), as well as provide an explicit opportunity for clinicians to adjust their action and coping plans according to the barriers and facilitators they have experienced thus far in their implementation. A booster session may be most effective during an active volitional phase, once clinicians have demonstrated strong intentions and some actions toward implementation, but may need continued support to persist with the delivery of the EBP $[91,92]$.

\section{Limitations}

This research yielded findings that speak to the utility of an individual implementation strategy based on the TPB. There were also limitations that should be addressed by future research. This study was focused on collecting preliminary outcome data and evaluating BASIS feasibility in a complex service delivery setting. By design, this study was unable to test hypotheses related to (1) sustainment of change in TPB mechanisms, (2) impact on additional implementation outcomes (e.g., EBP fidelity), (3) impact on youth mental health outcomes, and (4) mediation models evaluating mechanisms of change. Additionally, although data were collected, this study was unable to control for relevant organizational context factors (e.g., implementation climate) when testing prepost changes in proximal mechanisms due to power limitations. A fully powered randomized controlled trial of BASIS-potentially incorporating some of the revisions proposed above-could address these additional research questions within an effectiveness-implementation Hybrid Type 2 or Hybrid Type 3 design [93]. Considering the small sample size of this study, the findings presented should be interpreted with caution, especially for the few measure subscales that exhibited poor to moderate reliability. An emerging body of research, however, has demonstrated that Cronbach's alpha may underestimate the true value of reliability, especially in cases with small sample sizes and scales with few items [94]. Regardless, it will be important for future research with more participants and more complex research designs to reexamine the mechanisms proposed in the current study.

\section{Conclusions}

Existing implementation strategy compilations [36] contain few strategies explicitly designed to impact the individual-level mechanisms identified by the TPB (i.e., attitudes, social norms, perceived behavioral control, intentions). Further, most existing implementation strategies occur during the active implementation phase, rather than the preparation/pre-implementation phase.
BASIS is a novel, pragmatic implementation strategy designed to address TPB constructs prior to the initiation of active implementation activities, such as training and consultation. The current study suggests that BASIS was effective in shifting its theorized mechanisms of change, but additional work is needed to confirm these findings and to revise BASIS to enhance and sustain its effects so clinicians are more responsive to consultative supports. Continued development and testing of pragmatic strategies, such as BASIS, is critical to efficiently support large-scale EBP implementation in the pursuit of promoting better patient outcomes.

\section{Additional file}

Additional file 1: Detailed Measures Table. (DOCX 18 kb)

Abbreviations

AC: Attention control; BASIS: Beliefs and Attitudes for Successful Implementation in Schools; CBITS: Cognitive Behavioral Intervention for Trauma in Schools; EBP: Evidence-based practice; EBPAS: Evidence-Based Practice Attitudes Scale; HAPA: Health adaption process approach;

SICBS: School Implementation Citizenship Behavior Scale; TPB: Theory of planned behavior

\section{Acknowledgments}

We thank Kristine Lee and Elissa Picozzi for assisting with references and formatting.

\section{Funding}

This study and publication were supported, in part, by funding from the National Institute of Mental Health (R21MH108714), awarded to the first and second authors (Lyon \& Cook).

Availability of data and materials

Please contact the lead author for more information.

\section{Authors' contributions}

ARL and CRC conceptualized and co-led the full project. CRC, MTD, and ARL developed the revised BASIS implementation strategy. ARL drafted the initial manuscript outline and wrote the introduction. SN and SC coordinated the study, consented participants, and cleaned and summarized data. SN and MDP conducted analyses and drafted the results section. SKB and LMG drafted significant components of the manuscript discussion. All authors read and approved the final manuscript.

Ethics approval and consent to participate

All participants completed the study consent form. Study procedures and materials including the consent form were submitted by the second author and approved by the University of Washington Institutional Review Board on July 22, 2016. All study staff have been trained in ethical conduct of human subjects research.

Consent for publication

Not applicable.

Competing interests

The authors declare that they have no competing interests.

\section{Publisher's Note}

Springer Nature remains neutral with regard to jurisdictional claims in published maps and institutional affiliations. 


\section{Author details}

'University of Washington, 6200 NE 74th Street, Suite 100, Seattle, WA 98115, USA. ${ }^{2}$ University of Minnesota, 250 Education Sciences Bldg, 56 East River Road, Minneapolis, MN 55455, USA. ${ }^{3}$ Committee for Children, 2815 2nd Ave \#400, Seattle, WA 98121, USA.

Received: 31 December 2018 Accepted: 16 May 2019

Published online: 30 May 2019

\section{References}

1. Powell BJ, Beidas RS, Lewis CC, Aarons GA, McMillen JC, Proctor EK, et al. Methods to improve the selection and tailoring of implementation strategies. J Behav Health Serv Res. 2017;44:177-94.

2. Proctor $E_{1}$ Powell B, Mcmillen J. Implementation strategies: recommendations for specifying and reporting. Implement Sci. 2013:8:139.

3. Powell BJ, McMillen JC, Proctor EK, Carpenter CR, Griffey RT, Bunger AC, et al. A compilation of strategies for implementing clinical innovations in health and mental health. Med Care Res Rev. 2012;69:123-57.

4. Krause J, Van Lieshout J, Klomp R, Huntink E, Aakhus E, Flottorp S, et al. Identifying determinants of care for tailoring implementation in chronic diseases: an evaluation of different methods. Implement Sci. 2014;9:102.

5. Lewis CC, Puspitasari A, Boyd MR, Scott K, Marriott BR, Hoffman M, et al. Implementing measurement based care in community mental health: a description of tailored and standardized methods. BMC Res Notes. 2018;11:76.

6. Aarons GA, Hurlburt M, Horwitz SM. Advancing a conceptual model of evidence-based practice implementation in public service sectors. Admin Pol Ment Health. 2011;38:4-23.

7. Beidas RS, Kendall PC. Training therapists in evidence-based practice: a critical review of studies from a systems-contextual perspective. Clin Psychol Sci Pract. 2010;17:1-30.

8. Brackett MA, Reyes MR, Rivers SE, Elbertson NA, Salovey P. Assessing teachers' beliefs about social and emotional learning. J Psychoeduc Assess. 2012;30:219-36.

9. Dart EH, Cook CR, Collins TA, Gresham FM, Chenier JS. Test driving interventions to increase treatment integrity and student outcomes. Sch Psychol Rev. 2012;41:467-81.

10. Kincaid D, Childs K, Blase KA, Wallace F. Identifying barriers and facilitators in implementing schoolwide positive behavior support. J Posit Behav Interv. 2007:9:174-84.

11. Locke J, Lawson GM, Beidas RS, Aarons GA, Xie M, Lyon AR, Stahmer A, Seidman M, Frederick L, Oh C, Spaulding C. Individual and organizational factors that affect implementation of evidence-based practices for children with autism in public schools: a cross-sectional observational study. Implementation Science. 2019:14(1):29.

12. Aarons GA, Ehrhart MG, Farahnak LR, Hurlburt MS. Leadership and organizational change for implementation (LOCI): a randomized mixed method pilot study of a leadership and organization development intervention for evidence-based practice implementation. Implement Sci. 2015;10:11.

13. Glisson C, Schoenwald SK. The ARC organizational and community intervention strategy for implementing evidence-based children's mental health treatments. Ment Health Serv Res. 2005;7:243-59.

14. Glisson C. The organizational context of children's mental health services. Clin Child Fam Psychol Rev. 2002;5:233-53.

15. Michie S, Van Stralen M, West R. The behaviour change wheel: a new method for characterising and designing behaviour change interventions. Implement Sci. 2011;6:1.

16. Lewis CC, Boyd MR, Walsh-Bailey C, Lyon AR, Beidas R, Mittman B, et al. A systematic review of empirical studies examining mechanisms of dissemination and implementation in health. under review

17. Williams NJ. Multilevel mechanisms of implementation strategies in menta health: integrating theory, research, and practice. Adm Policy Ment Health. 2016:43:783-98.

18. Ajzen I. The theory of planned behaviour. Organ Behav Hum Decis Process. 1991;50:179-211.

19. Ajzen I. From intentions to actions: a theory of planned behavior. In: Kuhl J, Beckmann J, editors. Action control: from cognition to behavior. Berlin: Springer Berlin Heidelberg; 1985. p. 11-39. Available from: https://doi.org/10, 1007/978-3-642-69746-3_2.

20. Fishbein M, Ajzen I. Belief, attitude, intention, and behavior: an introduction to theory and research. New York: Addison-Wesley Pub. Co; 1975.
21. Eccles MP, Grimshaw JM, Johnston M, Steen N, Pitts NB, Thomas R, et al. Applying psychological theories to evidence-based clinical practice: identifying factors predictive of managing upper respiratory tract infections without antibiotics. Implement Sci. 2007;2:26.

22. Godin G, Bélanger-Gravel A, Eccles M, Grimshaw J. Healthcare professionals' intentions and behaviours: a systematic review of studies based on social cognitive theories. Implement Sci. 2008;3:36.

23. Ajzen I, Manstead A. Changing health-related behaviours: an approach based on the theory of planned behaviour. In: Hewstone M, Schut H, de Wit J, Van Den Bos K, Stroebe M, editors. The scope of social psychology: theory and applications. New York City: Psychology Press; 2007. p. 43-63.

24. Steinmetz H, Knappstein M, Ajzen I, Schmidt P, Kabst R. How effective are behavior change interventions based on the theory of planned behavior? Z Psychol. 2016;224:216-33.

25. Eccles MP, Hrisos S, Francis J, Kaner EF, Dickinson HO, Beyer F, et al. Do selfreported intentions predict clinicians' behaviour: a systematic review. Implement Sci. 2006;1:28.

26. Perou R, Bitsko RH, Blumberg SJ, Pastor P, Ghandour RM, Gfroerer JC, et al. Mental health surveillance among children-United States, 2005-2011. MMWR Suppl. 2013;62:1-35.

27. Farmer EMZ, Burns BJ, Phillips SD, Angold A, Costello EJ. Pathways into and through mental health services for children and adolescents. Psychiatr Serv. 2003:54:60-6.

28. Flaherty LT, Osher D. History of school-based mental health services in the United States. In: Weist MD, Evans S, Lever NA, editors. Handbook of School Mental Health Advancing Practice and Research. Boston: Springer; 2003. p. 11-22.

29. Rones M, Hoagwood K. School-based mental health services: a research review. Clin Child Fam Psychol Rev. 2000;3:223-41.

30. Teich $J$ L, Robinson G, Weist MD. What kinds of mental health services do public schools in the United States provide? Adv School Ment Health Promot. 2008;1:13-22.

31. Ennett ST, Ringwalt CL, Thorne J, Rohrbach LA, Vincus A, Simons-Rudolph A, et al. A comparison of current practice in school-based substance use prevention programs with meta-analysis findings. Prev Sci. 2003:4:1-14.

32. Evans SW, Weist MD. Commentary: implementing empirically supported treatments in the schools: what are we asking? Clin Child Fam Psychol Rev. 2004;7:263-7.

33. Odom SL, McLean ME, Johnson LJ, LaMontagne MJ. Recommended practices in early childhood special education: validation and current use. J Early Interv. 1995;19:1-17.

34. Owens JS, Lyon AR, Brandt NE, Warner CM, Nadeem E, Spiel C, et al. Implementation science in school mental health: key constructs in a developing research agenda. Sch Ment Heal. 2014;6:99-111.

35. Fishman J, Beidas R, Reisinger E, Mandell DS. The utility of measuring intentions to use best practices: a longitudinal study among teachers supporting students with autism. J Sch Health. 2018:88:388-95.

36. Powell BJ, Waltz TJ, Chinman MJ, Damschroder LJ, Smith JL, Matthieu MM et al. A refined compilation of implementation strategies: results from the expert recommendations for implementing change (ERIC) project. Implement Sci. 2015:10:21.

37. Cook CR, Lyon AR, Kubergovic D, Wright DB, Zhang Y. A supportive beliefs intervention to facilitate the implementation of evidence-based practices within a multi-tiered system of supports. Sch Ment Heal. 2015;7:49-60.

38. Duong MT, Cook CR, Lee K, Davis C, Vazquez-Colon C, Lyon AR. Stakeholder perspectives on the development of a blended pre-implementation strategy for school-based behavioral health providers. under review;

39. Jaycox LH, Kataoka SH, Stein BD, Langley AK, Wong M. Cognitive behavioral intervention for trauma in schools. J Appl Sch Psychol. 2012;28:239-55.

40. Chafouleas SM, Johnson AH, Overstreet S, Santos NM. Toward a blueprint for trauma-informed service delivery in schools. Sch Ment Heal. 2016:8:144-62.

41. Overstreet S, Chafouleas SM. Trauma-informed schools: introduction to the special issue. Sch Ment Heal. 2016:8:1-6.

42. Nadeem E, Saldana L, Chapman J, Schaper H. A mixed methods study of the stages of implementation for an evidence-based trauma intervention in schools. Behavior therapy. 2018;49:509-24.

43. Aarons GA. Measuring provider attitudes toward evidence-based practice: consideration of organizational context and individual differences. Child Adolesc Psychiatr Clin N Am. 2005:14:255-viii.

44. Perkins HW, Meilman PW, Leichliter JS, Cashin MA, Presley CA. Misperceptions of the norms for the frequency of alcohol and other drug use on college campuses. J Am Coll Heal. 1999;47:253-8. 
45. Perkins HW. The contextual effect of secular norms on religiosity as moderator of student alcohol and other drug use. Research in the social scientific study of religion. 1994;6:187-208.

46. Chassin L, Presson CC, Sherman SJ, C E, Olshavsky RW. Predicting the onset of cigarette smoking in adolescents: a longitudinal study. J Appl Soc Psychol. 1984;14:224-43.

47. Berkowitz AD. Applications of social norms theory to other health and social justice issues. In: Perkins HW, editor. The social norms approach to preventing school and college age substance abuse: a handbook for educators, counselors, clinicians. San Francisco: Jossey-Bass; 2002.

48. Cioffi D, Garner R. On doing the decision: effects of active versus passive choice on commitment and self-perception. Personal Soc Psychol Bull. 1996:22:133-47.

49. Petrova PK, Cialdini RB, Sills SJ. Consistency-based compliance across cultures. J Exp Soc Psychol. 2007;43:104-11.

50. Miller WR, Rollnick S. Motivational interviewing: preparing people for change. 2nd ed. New York: Guilford Publications, Inc; 2002.

51. Boyd-Ball AJ, Dishion TJ. Family-centered treatment for American Indian adolescent substance abuse: toward a culturally and historically informed strategy. In: Liddle H, Rowe C, editors. Treating adolescent substance abuse: state of the science. New York: Cambridge; 2006. p. 423-48.

52. Connell AM, Dishion TJ, Yasui M, Kavanagh K. An adaptive approach to family intervention: linking engagement in family-centered intervention to reductions in adolescent problem behavior. J Consult Clin Psychol. 2007;75:568-79.

53. Dishion TJ, Kavanagh K. Intervening in adolescent problem behavior: a family-centered approach. New York: Guilford Press; 2003.

54. Lundahl BW, Kunz C, Brownell C, Tollefson D, Burke BL. A meta-analysis of motivational interviewing: twenty-five years of empirical studies. Res Soc Work Pract. 2010;20:137-60.

55. Frey AJ, Lee J, Small JW, Seeley JR, Walker HM, Feil EG. The motivational interviewing navigation guide: a process for enhancing teachers' motivation to adopt and implement school-based interventions. Adv School Ment Health Promot. 2013;6:158-73.

56. Reinke WM, Herman KC, Sprick R. In: Riley-Tillman TC, editor. Motivational interviewing for effective classroom management: the classroom check-up. New York: Guilford Press; 2011.

57. Walton GM, Cohen GL. A brief social-belonging intervention improves academic and health outcomes of minority students. Science. 2011;331:1447-51.

58. Stein BD, Kataoka S, Jaycox L, Steiger M, Wong M, Flink A, et al. The mental health for immigrants program: program design and participatory research in the real world. Handbook of school mental health: advancing practice and research. New York: Kluwer/Plenum; 2003. p. 179-90.

59. Stein BD, Jaycox LH, Kataoka SH, Wong M, Tu W, Elliott MN, et al. A mental health intervention for schoolchildren exposed to violence: a randomized controlled trial. JAMA. 2003;290:603-11.

60. Ngo V, Langley A, Kataoka SH, Nadeem E, Escudero P, Stein BD. Providing evidence-based practice to ethnically diverse youths: examples from the cognitive behavioral intervention for trauma in schools (CBITS) program. J Am Acad Child Adolesc Psychiatry. 2008;47:858-62.

61. Vona P, Wilmoth P, Jaycox LH, McMillen JS, Kataoka SH, Wong M, et al. A webbased platform to support an evidence-based mental health intervention: lessons from the CBITS web site. Psychiatr Serv. 2014;65:1381-4.

62. Herschell AD, Kolko DJ, Baumann BL, Davis AC. The role of therapist training in the implementation of psychosocial treatments: a review and critique with recommendations is. Clin Psychol Rev. 2010:30:448-66.

63. Cook CR, Davis C, Brown EC, Locke J, Ehrhart MG, Aarons GA, Larson M, Lyon AR. Confirmatory factor analysis of the Evidence-Based Practice Attitudes Scale with school-based behavioral health consultants. Implementation Science. 2018(1):116.

64. Aarons GA. Mental health provider attitudes toward adoption of evidencebased practice: the evidence-based practice attitude scale (EBPAS). Mental health services research, vol. 6; 2004. p. 61-74.

65. Ajzen I. Constructing a TPB questionnaire: conceptual and methodological considerations; 2006

66. Francis J, Eccles MP, Johnston M, Walker AE, Grimshaw JM, Foy R, et al. Constructing questionnaires based on the theory of planned behaviour: a manual for health services researchers [internet]. 2004. Available from: http://openaccess.city.ac.uk/1735/. [cited 2018 May 25]

67. Palinkas LA, Aarons GA, Horwitz S, Chamberlain P, Hurlburt M, Landsverk J. Mixed method designs in implementation research. Administration and policy in mental health, vol. 38; 2011. p. 44-53.
68. Lyon AR, Cook CR, Brown EC, Locke J, Davis C, Ehrhart M, et al. Assessing organizational implementation context in the education sector: confirmatory factor analysis of measures of implementation leadership, climate, and citizenship. Implement Sci. 2018;13:5.

69. Ehrhart MG, Aarons GA, Farahnak LR. Going above and beyond for implementation: the development and validity testing of the implementation citizenship behavior scale (ICBS). Implement Sci. 2015;10:65.

70. Kortteisto T, Kaila M, Komulainen J, Mäntyranta T, Rissanen P. Healthcare professionals' intentions to use clinical guidelines: a survey using the theory of planned behaviour. Implement Sci. 2010;5:51.

71. Ehrhart MG, Aarons GA, Farahnak LR. Assessing the organizational context for EBP implementation: the development and validity testing of the implementation climate scale (ICS). Implement Sci. 2014;9:157.

72. Yarnold P. ODA vs. $\pi$ and K: paradoxes of kappa. Optimal Data Anal. 2016;5: 160-1.

73. Tomasone JR, Martin Ginis KA, Estabrooks PA, Domenicucci L'. Changing minds': determining the effectiveness and key ingredients of an educational intervention to enhance healthcare professionals' intentions to prescribe physical activity to patients with physical disabilities. Implement Sci. 2014;9:30.

74. Armitage CJ, Conner M. Efficacy of the theory of planned behaviour: a meta-analytic review. Br J Soc Psychol. 2001:40:471-99.

75. Sparks P, Shepherd R, Wieringa N, Zimmermanns N. Perceived behavioural control, unrealistic optimism and dietary change: an exploratory study. Appetite. 1995;24:243-55.

76. White KM, Smith JR, Terry DJ, Greenslade JH, McKimmie BM. Social influence in the theory of planned behaviour: the role of descriptive, injunctive, and in-group norms. Br J Soc Psychol. 2009;48:135-58.

77. Lyon AR, Stirman SW, Kerns SEU, Bruns EJ. Developing the mental health workforce: review and application of training approaches from multiple disciplines. Admin Pol Ment Health. 2010;38:238-53.

78. Aarons GA, Ehrhart MG, Farahnak LR. The implementation leadership scale (ILS): development of a brief measure of unit level implementation leadership. Implement Sci. 2014;9:45.

79. Beidas RS, Edmunds J, Ditty M, Watkins J, Walsh L, Marcus S, et al. Are inner context factors related to implementation outcomes in cognitive-behavioral therapy for youth anxiety? Admin Pol Ment Health. 2014;41:788-99.

80. Lyon AR, Pullmann MD, Walker SC, D'Angelo G. Community-sourced intervention programs: Review of submissions in response to a statewide call for "promising practices". Admin Pol Ment Health. 2017;44(1):16-28.

81. Helfrich CD, Kohn MJ, Stapleton A, Allen CL, Hammerback KE, Chan KCG, et al. Readiness to change over time: change commitment and change efficacy in a workplace health-promotion trial. Front Public Health. 2018:6.

82. Schwarzer R. Modeling health behavior change: how to predict and modify the adoption and maintenance of health behaviors. Appl Psychol. 2008:57:1-29.

83. Schwarzer R, Lippke S, Luszczynska A. Mechanisms of health behavior change in persons with chronic illness or disability: the health action process approach (HAPA). Rehabil Psychol. 2011;56:161-70.

84. Potthoff S, Presseau J, Sniehotta FF, Johnston M, Elovainio M, Avery L. Planning to be routine: habit as a mediator of the planning-behaviour relationship in healthcare professionals. Implement Sci. 2017;12:24.

85. Barrett PM, Farrell LJ, Ollendick TH, Dadds M. Long-term outcomes of an Australian universal prevention trial of anxiety and depression symptoms in children and youth: an evaluation of the friends program. J Clin Child Adolesc Psychol. 2006;35:403-11.

86. Bradshaw C, Reinke W, Brown L, Bevans K, Leaf P. Implementation of schoolwide positive behavioral interventions and supports (PBIS) in elementary schools: observations from a randomized trial. Educ Treat Child. 2008;31:1-26.

87. Hagger MS, Luszczynska A. Implementation intention and action planning interventions in health contexts: state of the research and proposals for the way forward. Appl Psychol. 2014;6:1-47.

88. Masia-Warner C, Klein RG, Dent HC, Fisher PH, Alvir J, Albano AM, et al. School-based intervention for adolescents with social anxiety disorder: results of a controlled study. J Abnorm Child Psychol. 2005;33:707-22.

89. Dijkstra M, Mesters I, De Vries H, van Breukelen G, Parcel GS. Effectiveness of a social influence approach and boosters to smoking prevention. Health Educ Res. 1999;14:791-802.

90. Tolan PH, Gorman-Smith D, Henry D, Schoeny M. The benefits of booster interventions: evidence from a family-focused prevention program. Prev Sci. 2009;10:287-97.

91. Aliabad HO, Vafaeinasab M, Morowatisharifabad MA, Afshani SA, Firoozabadi MG, Forouzannia SK. Maintenance of physical activity and exercise capacity 
after rehabilitation in coronary heart disease: a randomized controlled trial. Glob J Health Sci. 2014;6:198-208.

92. Fleig L, Pomp S, Schwarzer R, Lippke S. Promoting exercise maintenance: how interventions with booster sessions improve long-term rehabilitation outcomes. Rehabil Psychol. 2013;58:323-33.

93. Curran GM, Bauer M, Mittman B, Pyne JM, Stetler C. Effectivenessimplementation hybrid designs: combining elements of clinical effectiveness and implementation research to enhance public health impact. Med Care. 2012;50:217-26.

94. McNeish D. Thanks coefficient alpha, we'll take it from here. Psychol Methods. 2018:23:412-33.

Ready to submit your research? Choose BMC and benefit from:

- fast, convenient online submission

- thorough peer review by experienced researchers in your field

- rapid publication on acceptance

- support for research data, including large and complex data types

- gold Open Access which fosters wider collaboration and increased citations

- maximum visibility for your research: over $100 \mathrm{M}$ website views per year

At $\mathrm{BMC}$, research is always in progress.

Learn more biomedcentral.com/submissions 